\title{
Preparation Strategies and Solid-State NMR Characterization of Supramolecular Architectures based on Halogen Bonds, Chalcogen Bonds, and Pnictogen Bonds
}

\author{
David L. Bryce \\ University of Ottawa \\ dbryce@uottawa.ca
}

In this talk, I will describe our recent work on the design and preparation of novel supramolecular architectures based on a range of element-based non-covalent interactions such as halogen bonds, chalcogen bonds, and pnictogen bonds. In addition to standard wet chemistry and slow evaporation methods, the utility of mechanochemical and cosublimation techniques will be discussed. Considered together, these methods enable a broad exploration of the polymorphic cocrystalline landscape. For example, the cosublimation approach overcomes an anticooperative halogen-bonding effect to produce fully saturated cocrystals of the tritopic halogen bond donor 1,3,5-trifluoro-2,4,6-triiodobenzene with 1,4-diazabicyclo[2.2.2] octane. $^{1} \quad$ I also report on dicyanoselenodiazole and dicyanotelluradiazole derivatives which work as promising supramolecular synthons with the ability to form double chalcogen bonds with a wide range of electron donors including halides and oxygen- and nitrogen-containing heterocycles. ${ }^{2}$ In addition to X-ray diffraction, solid-state multinuclear magnetic resonance (SSNMR) and nuclear quadrupolar resonance (NQR) spectroscopies are employed to characterize all products and to establish spectral signatures for the various classes of bonds. Given the elements involved in these bonds, we report on a wide range of nuclides including e.g., ${ }^{17} \mathrm{O},{ }^{31} \mathrm{P},{ }^{35 / 37} \mathrm{Cl},{ }^{77} \mathrm{Se},{ }^{79 / 81} \mathrm{Br},{ }^{125} \mathrm{Te},{ }^{127} \mathrm{I}$, ${ }^{121 / 123} \mathrm{Sb}$, etc. As most of these are quadrupolar nuclides, the utility of specialized NMR techniques and very high applied magnetic fields will be discussed. In favourable cases, in-situ solid-state NMR spectroscopy allows for real-time monitoring of cocrystallization reactions and for the determination of activation energies. ${ }^{3}$

1. Szell, P. M. J.; Gabriel, S. A.; Caron-Poulin, E.; Jeannin, O.; Fourmigué, M.; Bryce, D. L. Cryst. Growth Des. $2018,18,6227$. https://doi.org/10.1021/acs.cgd.8b01089

2. Kumar, V.; Xu, Y.; Bryce, D. L. Chem. Eur. J. 2020, 26, 3275-3286. https://doi.org/10.1002/chem.201904795

3. Xu, Y.; Champion, L.; Gabidullin, B.; Bryce, D. L. Chem. Commun. 2017, 53, 9930. https://doi.org/10.1039/C7CC05051H

Keywords: cocrystal, NMR, solid-state NMR, halogen bond, chalcogen bond 